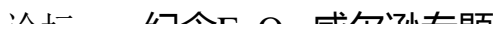 \\ E. O. 威尔逊与岛屿生物地理学理论
}

\author{
阎恩荣*，斯幸峰@，张健，陈小勇 \\ 华东师范大学生态与环境科学学院, 上海 200241
}

阎恩荣, 斯幸峰, 张健, 陈小勇 (2022) E. O. 威尔逊与岛屿生物地理学理论. 生物多样性, 30, 22024. doi: 10.17520/biods.2022024.

Yan ER, Si XF, Zhang J, Chen XY (2022) Edward O. Wilson and the Theory of Island Biogeography. Biodiversity Science, 30, 22024. doi: $10.17520 /$ biods.2022024.

\section{Edward O. Wilson and the Theory of Island Biogeography}

Enrong Yan ${ }^{\mathbb{D} *}$, Xingfeng $\mathrm{Si}^{\circledR}$, Jian Zhang ${ }^{\circledR}$, Xiaoyong Chen ${ }^{\mathbb{D}}$

School of Ecological and Environmental Sciences, East China Normal University, Shanghai 200241

20 世纪伟大的博物学家和生物地理学家爱德 华. 奥斯本. 威尔逊 (Edward Osborne Wilson, 1929-2021) 与杰出的理论和数学生态学家罗伯 特·赫尔默·麦克阿瑟 (Robert Helmer MacArthur, 1930-1972)共同开创的岛屿生物地理学理论是他们 青年时代的巅峰之作。但是, 由于威尔逊后来在昆 虫学和社会生物学等领域的巨大成就, 加之麦克阿 瑟的英年早逝, 在很多介绍威尔逊学术成就的出版 物中, 往往没有详细交代他们在岛屿生物地理学理 论上的合作佳话。在威尔逊刚辞世之际, 回顾他在 岛屿生物地理学领域的宝贵科学遗产, 评述其对推 动科学革命的贡献, 一方面为了缅怀他勇敢探索、 富于创新和谦逊合作的优秀个人品质, 另一方面也 启迪我们要拥有勇于挑战学术高峰的科学精神。

岛屿生物地理学理论最早在Evolution期刊发表 (MacArthur \& Wilson, 1963), 随后又以专论形式系 统阐述(MacArthur \& Wilson, 1967)。他们的最终目 标是发展一个基于迁入、局部灭绝、物种形成等基 本生态和进化过程的动态生物地理学的一般理论, 用来解释各种各样的孤岛现象, 包括物种丰富度模 式、物种拓殖定居能力和生态位转换, 以及孤立生 物类群的进化。多年来, 尽管受到一些批评, 岛屿
生物地理学理论为理解生物多样性的形成和维持 机制奠定了可预测的理论基础, 更开辟了20世纪中 期科学革命结构转变的新范式(从静态描述到动态 定量) (Kuhn, 1996), 从而为生物地理学、生态学和 进化生物学等提供了真正具有变革性的见解。

以下，我们简要回顾威尔逊的物种进化知识启 蒙教育、南太平洋岛屿考察、生物类群循环理论 (theory of taxon cycles)、岛屿生物地理学理论及其 控制实验验证, 最后, 简单总结岛屿生物地理学理 论的科学价值。

\section{物种扩散和进化知识的启蒙}

威尔逊成为一名科学家的动力源于对自然界 中生物(包括其毕生研究的蚂蚁类群)不可抗拒的热 情。他一开始对鸟类感兴趣, 后来右眼在钓鱼时不 幸误伤失明, 无法远视, 就转向研究昆虫。正如达 尔文的自然选择理论受益于亚历山大.冯.洪堡 (Alexander von Humboldt)、詹姆斯·赫顿 (James Hutton)、查尔斯·莱伊尔(Charles Lyell)、托马斯·罗 伯特.马尔萨斯(Thomas Robert Malthus)等那个时代 其他有远见的科学家一样, 威尔逊攻读研究生时 (1950年)就对古生物学家威廉·迪勒·马修(William 
Diller Matthew)提出的北温带是新生代物种起源中 心(Matthew，1915), 以及后来菲利普·达林顿(Philip Darlington)将物种起源中心确定为旧世界热带地区 (Darlington, 1948)的学术观点产生浓厚兴趣。据威 尔逊回忆，马修和达林顿这史诗般的全球疆域的生 物地理学观点一直刻在他的脑海中, 极大地启发了 他的科学想象力(Wilson, 1994)。

与大多数博物学家类似, 威尔逊也选择新世界 热带地区开始自己的职业生涯。23岁时(1953年), 哈 佛大学的研究生奖学金资助了他在古巴和墨西哥 的科学考察。他最具影响力的导师之一达林顿是著 名的昆虫学家和动物地理学家, 同时也是他在哈佛 大学的同事。当他准备第一次热带采集之旅时, 达 林顿为这位前途无量的研究生提供了极具帮助的 建议。

\section{2 结缘南太平洋岛屿}

1954年3月, 达林顿向威尔逊提供了一个千载 难逢的机会一一去南太平洋的新几内亚群岛采集 蚂蚁标本, 他毫不犹豫地接受了这份礼物。正如威 尔逊在自传中所述(Wilson, 1994): “一百年前, 年轻 的华莱士在那里开始动物分布研究, 并将动物地理 学转化为一门科学学科, 而我也正在这个领域开始 工作。谁知道这段经历会如何改变我成为一个动物 地理学家的想法?”

1954年11月24日, 威尔逊离开波士顿开始了 10 个月的南太平洋远行。12月, 先在斐济的维提拉武 岛采集到了几种当地特有的蚂蚁种类。短暂停留后, 前往美拉尼西亚群岛最南端的新喀里多尼亚, 这里 的动植物是在近数百万年间陆续由澳洲往东或是 由所罗门群岛经由新赫布里底群岛(New Hbrides) 往南迁移而来, 彼此交流, 进化出了独特的生态谱 系。在努美亚附近, 他采集的粗角蚁属(Cerapachys) 和束蚁属 (Sphinctomyrmex) 是人类首次采集到的蚂 蚁新物种。他发现, 粗角蚁属工蚁为了征服猎物, 以类似热带大陆地区的行军蚁的方式, 集体出猎。 行军蚁分布于亚洲和澳洲, 无法渡过珊瑚海(Coral Sea)到达新喀里多尼亚定居。粗角蚁属蚂蚁在此地 所采取的行军蚁生活方式, 或许是它们在这里如此 “成功”拥有优势地位, 而罕见于其他大陆的原因。 威尔逊当时对这个现象的描述和推测, 事后被证明
对于追踪蚂蚁进化过程非常重要。他在回忆录中写 道: “这个想法在当时并未成形, 之所以记录它们, 纯粹是因为兴趣。然而, 三年后在重新建立行军蚁 的进化起源时，这份野外记录派上了大用场” (Wilson, 1994)。

1955年1月初, 威尔逊到达新赫布里底群岛(现 在的瓦努阿图), 这里是前往西太平洋更偏远岛屿 的驿站, 向北, 由纯热带的所罗门群岛接收来自亚 洲的生物; 向南, 由亚热带的新喀里多尼亚接收来 自澳洲的生物。那时, 从来没有人在这里采集过蚂 蚁, 因此, 他的每一条记录都是新的发现。2月, 他 专门去西澳大利亚的埃斯佩兰斯(Esperance) 寻找最 原始的大眼响蚁(Nothomyrmecia macrops), 用来研 究蚂蚁的社会生活起源, 可惜没有找到。3月份, 转 战“最标准” 的热带地区新几内亚, 先在布朗河谷采 集到了 50 种蚂蚁, 其中很多是未知的新种; 之后, 在苏加利高原山脚的雨林, 找到了以集群形式寄生 在其他种类蚂蚁窝巢中的种类。

4 月, 他到达新几内亚东北胡安半岛 (Huon Peninsula)的沙拉威吉山脉(Sarawaget Range), 在这 个从未被昆虫学家造访过的大山里采集并研究蚂 蚁。这次山地之旅虽然在蚂蚁采集上没有什么特别 之处, 但在返程的路上, 威尔逊却在蚂蚁的分歧进 化问题上灵光乍现。他在思考新几内亚的蚂蚁分布 现象。虽然每公里间的森林外貌结构变化非常小, 但蚂蚁群落的结构变化却相当惊人。这些变异来源 有些是栖息地的根本变化, 但是, 许多变异并不容 易解释, 尤其是地理距离增加所造成的物种变化。 1958年, 他专门就此写了一篇文章(Wilson, 1994):

无论从比较生态学的哪一个层面来评价, 新几 内亚蚂蚁群落最大的特点莫过于物种超级丰 富。……除了单纯的数量外, 个别物种不一致的片 断分布增加了整个群落的复杂度。没有任何两个地 点具有完全相同的动物群落。想想看, 上百种蚂蚁 因此而发生进化，显然，整个新几内亚动物群落的 时空结构必定表现出万花筒般的面貌。这对个别蚂 蚁物种的进化所产生的效果一定是非常可观的，很 可能促进了局域种群在遗传方面的分歧进化....... 。

威尔逊非常高兴自己能在未经训练的采集和 长途考察中感悟出一个事后被证明应用广泛的生 态学模式, 也就是生物类群循环理论。完成新几内 
亚群岛考察后, 他在澳洲昆士兰雨林继续调查了一 周。之后, 在斯里兰卡寻找到了全世界最罕见的西 蒙原臭蚁(Aneuretus simoni)。至此, 热带岛屿的野外 工作结束。

\section{3 生物类群循环理论的提出}

对新几内亚和美拉尼西亚蚂蚁的考察研究, 从 根本上改变了威尔逊的科学生涯, 使其从一个传统 的系统学家和博物收藏家转变为一个非常有洞察 力和口才出众的生物地理学家、进化生物学家和生 物多样性保护者。从南太平洋岛屿回来不久, 他就 开始整理和发表考察成果, 其中, 有关生物类群循 环的两篇论文成为了他与麦克阿瑟关于物种平衡 理论的序篇。1959年, 威尔逊提出了初步的理论和 分析, 然后成功地将这一理论扩展为更全面、更完 整的岛屿生物历史和生态动态理论(Wilson, 1961)。

之前由达尔文和华莱士发展起来的起源-扩散-适 应中心 (the Center of Origin-Dispersal-Adaptation, CODA)思想的一个关键特征是物种和生物群落的优 势现象。Matthew (1915)、Simpson (1943) 和 Darlington (1948)假设, 从起源中心扩散出来的物 种会占据主导地位, 取代占据外围区域的物种。威 尔逊着迷于这些观点, 因此开始确定生物地理优势 的生物学本质。他把CODA的传统认知从全球和地 质尺度聚焦到局域尺度，如群岛和岛屿尺度。

事实上, 20 世纪 50 年代后期的生物地理学已具 备了这种革命性思想成熟的时机, 理论发展到极限, 积累了一堆例外和问题, 未能将观察到的格局与其 他学科结合起来。然而, 生态学、进化生物学和种 群生物学的新进展, 使这种综合成为可能。正如威 尔逊在阐述他的理论时所描述的那样, “普遍适应 的本质和主要生物迁移扩散背后的机制显然是现 代进化理论的重大问题之一。我们现在的知识可以 并且应该能对这些因果过程进行更精细的分析。”

威尔逊的理论确实是无与伦比的, 因为它具有 跨领域整合的能力, 解释和证明了一系列生物地理 模式和现象的因果联系。他总结道: “利用扩张、多 样化和收缩循环模式的科学假说, 可以解释最初扩 散之后的进化事件”。他推测, 大多数猛蚁(ponerine ants) 是按照Darlington (1957)所描述的“迁移格局形 式”，遵循“经典的过滤效应”, 从东南亚入侵新几内
亚, 再拓殖到美拉尼西亚(Wilson, 1959)。在岛屿和 群岛上, 物种组成的这种非常规律的衰减模式, 相 当于当代科学家所说的群落 “嵌套”, 在威尔逊所描 述的现象中, 这是一种由关键物种间迁移能力差异 所驱动的模式。

威尔逊将生物类群循环定义为 3 个阶段的进化 和生态变化序列。第一阶段的物种是那些在群岛或 区域具有相对连续地理分布范围的物种, 它们的性 状无地理差异。岛屿种群已适应大陆来源地的海滨 栖息地，因此易于扩散，并可预适应和拓殖定居于 具有类似栖息地特征的岛屿边缘。随后，这些拓殖 种群就会进入“进化陷阱” (Mayr, 1942; Darlington, 1957)，因为海滨栖息地不仅是边缘的，而且是短暂 的，容易受到演替或大规模风暴等灾难性事件的破 坏。然而，一些拓殖种群可能会通过适应和入侵美 拉尼西亚岛体内部的雨林, 从而逃离海滨生境, 并 与拓殖和祖先种群隔离, 最终为群岛种群的进化分 歧创造条件。如果进化分歧持续到后代种群中形成 新种的程度, 那么它们就进入生物类群循环的第二 阶段。这些岛屿特有种经历了性状更替和生态位转 化，因此变得越来越特化于岛屿内部生境。

如果分化和特化进展到这个程度，岛屿本土特 有种就容易在群岛内和整个群岛灭绝，从而进入生 物类群循环的第三阶段。曾经广布的岛内本土特有 种的地理范围缩小，成为局限于一两个岛屿的残余 种。威尔逊假设: “对大多数种群来说, 下一个也是 最后一个步骤是局域灭绝(整个群岛)，或极端特有 化，并可能因第一和第二阶段物种的入侵产生的新 竞争而加速。”他用非常清晰而又深刻的术语描述 了这一系列相互关联的进化和生态现象的驱动力: 种间相互作用。在海滨及其边缘和临时栖息地的不 同拓殖过程之后，种间相互作用驱动了岛内物种行 为、栖息地和地理分布的改变, 以及种群与特有种 的分化和灭绝。

值得注意的是，虽然威尔逊在两篇论文中都没 有使用“平衡”这个词，但他在1959年的论证中提出 了物种周转和自然平衡的概念, 并称之为“饱和” (saturation)。单岛动物群落接近岛屿大小所设定的 上限。可以推断，作为一种法则，只有在原有物种 灭绝为它们腾出空间的情况下, 新物种才可以入侵 一个岛屿。因此，威尔逊的饱和概念更类似于Lack 
(1937)的填满岛屿生态空间的想法, 而不是Munroe (1963)及后来麦克阿瑟和威尔逊的物种平衡概念, 后者没有上限, 而是代表了迁入、灭绝和成种率间 的动态平衡。

Wilson (1961) 通过描述物种饱和数量如何随岛 屿面积和隔离度的变化, 进一步论证了生物类群循 环的地理背景。他将种面积关系, 至少部分归因于 岛屿面积和物种灭绝间的反比关系。需要指出的是, 威尔逊对种面积关系的数学建模相对简单, 但基于 相同的公式, MacArthur和Wilson (1963)的幂函数或 双对数模型将其作为数学核心。无论如何, 威尔逊 确实观察到幂函数模型的幂在动物类群间和动物 分布区间的差异，他认为变异残差(在种面积关系 中分散)是由人为干扰导致的岛屿间栖息地差异造 成的, 或者仅仅是由于岛屿动物取样完整性不同导 致的。这就是威尔逊的初始理论的数学运算应用程 度, 两篇论文都没有提供描述循环过程平衡的数学 公式。

虽然生物类群循环理论是描述性的, 但其概念 框架的前景是不可否认的, 在科学革命中发挥了至 关重要的先驱作用, 为岛屿生物地理学提出了一个 引人注目的新范式, 超越了传统的岛屿生物地理学 静态理论, 证明了生态和进化过程是动态的、反复 的和相互关联的。

\section{4 与麦克阿瑟的第一次合作: 岛屿物 衡理论}

1961年, 在纽约举行的美国科学促进会会议上, 拉里·斯洛博金(Larry Slobodkin)将麦克阿瑟(两人都 是哈钦森的研究生)介绍给威尔逊。威尔逊和麦克阿 瑟有很多共同点, 包括对博物学和生态学的热爱, 以及对生物多样性和地理普适性解释的渴望 (Wilson 1994)。威尔逊觉得生物地理学处于“大混 乱”状态, “新思想的时机已经成熟”, 就给麦克阿瑟 分享他关于岛屿、生物类群循环和自然平衡的想 法。麦克阿瑟对生物地理学也很着迷, 他很快同意 与威尔逊合作, 用自己的概念综合和数学抽象能力 补充威尔逊的理论结构。

他们第一次合作的目标在论文最后一段有明 确的说明: “处理可能适用于其他动物的通用平衡 准则, 以及平衡状态的一些生物学含义”
(MacArthur \& Wilson, 1963)。这确实是一个非常恰 当的模型描述。无疑，他们的模型具备实现已经酝 酿了几十年的科学革命的潜力。但他们认为, 首先 有必要在一篇严谨的论文中阐明他们模型的基本 前提，因此就促成了他们第一篇合作论文在 Evolution发表。

但是，这篇具有革命性的论文的战略意义有 限。虽然其隐含目标是进一步从定性和定量两方面 发展出生物类群循环理论，但他们首次合作关注的 仅是鸟类群落的物种丰富度格局及其平衡成因, 或 如他们所说，迁入、灭绝和成种等反复过程间“非常 动态的平衡”。事实上，他们在第一次合作中过于保 守，以至于将推论局限于一个特定的类群一一印度 一澳洲岛屿鸟类群落。除了有限的生物类别，显然， 包括群岛内和跨群岛的物种组成(即镶嵌分布和群 落嵌套)、性状和生态位转化、生态释放，以及岛内 和岛间种群局域特化等，都是超出这篇论文以外的 有趣格局。

正如他们在摘要中所述, 该论文的主要目的是 “表达平衡状态的标准和含义，而不是将它们扩展 到印度澳洲鸟类群落之外。”威尔逊和麦克阿瑟都是 杰出的博物学家，他们之前的研究清楚说明了种间 差异和相互作用在塑造岛屿地理格局方面的重要 性。然而, 在他们的模型中, 物种被视为“黑箱”。他 们强调: 我们知道它们是不同的, 但在动态平衡的 假设下, 这些差异可能不是解释物种丰富度格局的 必要条件。

他们构建的物种中性模型 (species-neutral model) 是革命性的。这一成功很大程度上源于麦克 阿瑟和威尔逊采用图形和数学互补形式巧妙地解 释了两物种丰富度的普适模式。对大多数科学家来 说，由此产生的模型既容易理解又易于解释，而且 经得起逻辑推敲和可证伪预测的考验。例如，他们 不仅证明了动态平衡如何解释物种-面积关系和物 种一隔离度关系，而且还证明了为什么尽管物种组 成发生了变化, 物种丰富度仍然相对稳定。此外, 他们证明了如何采用物种中性模型的数学形式估 计类群平均扩散距离等种群性质, 动物多样性平衡 被打破(例如风期风或火山爆发)后岛屿物种饱和度的 动态, 以及再次达到均衡所需的时间。模型的图形 形式还能够发展出一套关于岛屿群落生物地理动态 
(例如迁入、灭绝和周转率应随面积、隔离度、气候 以及源区物种数量而变化)的定性和可证伪的预测, 这对他们理论的评估和未来发展同样重要。

为了证明物种平衡理论, 他们从威尔逊的物种 适应区视角, 利用Dammermann (1948)关于1883年 火山爆发后喀拉喀托 (Krakatau) 鸟类动态的详细记 录, 在动态平衡背景下, 计算并检验他们的理论预 测。结果发现, 到1933年, 喀拉喀托已被6种广布的 本地猛蚁所拓殖。喀拉喀托火山的案例很好地证明 了岛屿物种通过快速而反复的拓殖和积累, 群落趋 于饱和, 最终灭绝平衡了迁入一这就形成了生物 类群循环的中心前提, 成就了岛屿生物地理学的平 衡理论。

喀拉喀托火山的事实也令人信服地证明了借 助天然事件开展实验的效用, 同时, 也为巧妙设计 岛屿生物地理学野外人工控制实验播下了创造性 的种子, 包括由威尔逊和他的研究生丹尼尔. 辛伯 洛夫(Daniel Simberloff)所设计的经典的红树林岛礁 节肢动物去除实验。

\section{5 与麦克阿瑟的第二次合作: 岛屿生物胡 理学理论}

1963年论文发表后，威尔逊与麦克阿瑟成了亲 密朋友。1964年12月, 他们决定写一本关于岛屿生 物地理学的全面书籍, 目的是创造新的模型, 并将 他们的理论模型扩展到尽可能多的生态学领域。3 年后，他们的跨时代著作 The Theory of Island Biogeography出版。威尔逊后来在评价他们专著的 成功时说: “麦克阿瑟和我完成了大部分我们要做 的事情。以种群生物学为基础, 我们开始统一, 或 至少开始统一生物地理学和生态学。”

这本专著是自达尔文和华莱士以来最全面的 生物地理学理论著作。除了两章导论和发展平衡理 论的第 3 章, 其他 5 章探讨了广泛的主题, 包括: $r / k$ 选择及其与进化的相关性, 以及岛屿种群的建立 (第4章); 种群统计学、种间相互作用和生态位动态 (第5章); 扩散曲线、群岛几何学和生物交换(第6章); 以及拓殖定居和适应性扩散之后的进化变化(第7 章)。在专著中, 平衡模型最具革命性的特征, 但他 们的目标远比这个更宏大、更全面。在该书出版之 前, 他们给当时在密歇根大学的斯洛博金寄送了一
份草稿和注释, 提出了他们的最终目标, 那就是, 不仅改变岛屿生物地理学, 而且改变整个生物地理 学领域(Lomolino \& Brown, 2009)。

与威尔逊关于生物类群循环的原始论文形成 鲜明对比的是, 专著 The Theory of Island Biogeography没有包括一幅物种分布图。可以猜想, 这是有意而为, 重在强调普适性和综合价值, 而不 是过分描述和解释特殊细节。不幸的是, 他们混淆 了最终目标, 在发展普适理论时, 仍以物种和过程 为基础, 即物种生物地理学的基本过程(如迁入、灭 绝和成种)有何不同。因此, 他们在结论中回到了非 常普适的主题, 呼吁生物地理学领域“按照种群生 态学和遗传学的首要原则重新制定......暂时不关 注传统的高等类群分布和地质变化的作用问 题......转而详细研究选定物种。物种的生物地理学 需要理论和实验, 而这些理论和实验在很大程度上 必须是新颖的”。

需要指出的是, 不管我们如何评价这本著作的 成功或不足, 它无疑对岛屿生物地理学以及生态学 和进化生物学等都产生了革命性的影响(图1)。威尔 逊后来也很欣慰地总结道: “岛屿生物地理学理论 经历了 40 余年的考验，被证明总体上是正确的” (Wilson, 2009)。虽然岛屿生物地理学理论对于最大 和最复杂的系统来说仍然解释力有限, 但已经发挥 了足够的作用, 成为生态学和生物地理学的重要组 成部分, 也是保护生物学的基石。

\section{6 寻找岛山与生物地理学理论的实验证据}

虽然岛屿生物地理学构想很好地按照他们设 定的目标发展, 但终归是停留在书本上和口头上的 理论。威尔逊是一个忠实的野外生态学家, 他非常 渴望在现实的岛屿上检验他们的新理论。但是, 由 于路途的遥远，回到美拉尼西亚是不可能的。于是, 就产生了在离家近的地方建立海岛生物学自然实 验室的想法。在这个梦想的诱惑下, 威尔逊很快想 到佛罗里达群岛是一个合乎逻辑的去处, 因为有众 多的岛礁可供选择。

威尔逊认为, 研究实验岛屿生物地理学最好的 方法是从许多生态相似但面积和距离不同的小岛 开始, 然后把它们变成微型的喀拉喀托火山。也就 是说, 找到一种方法来根除动物群落, 然后跟踪物 


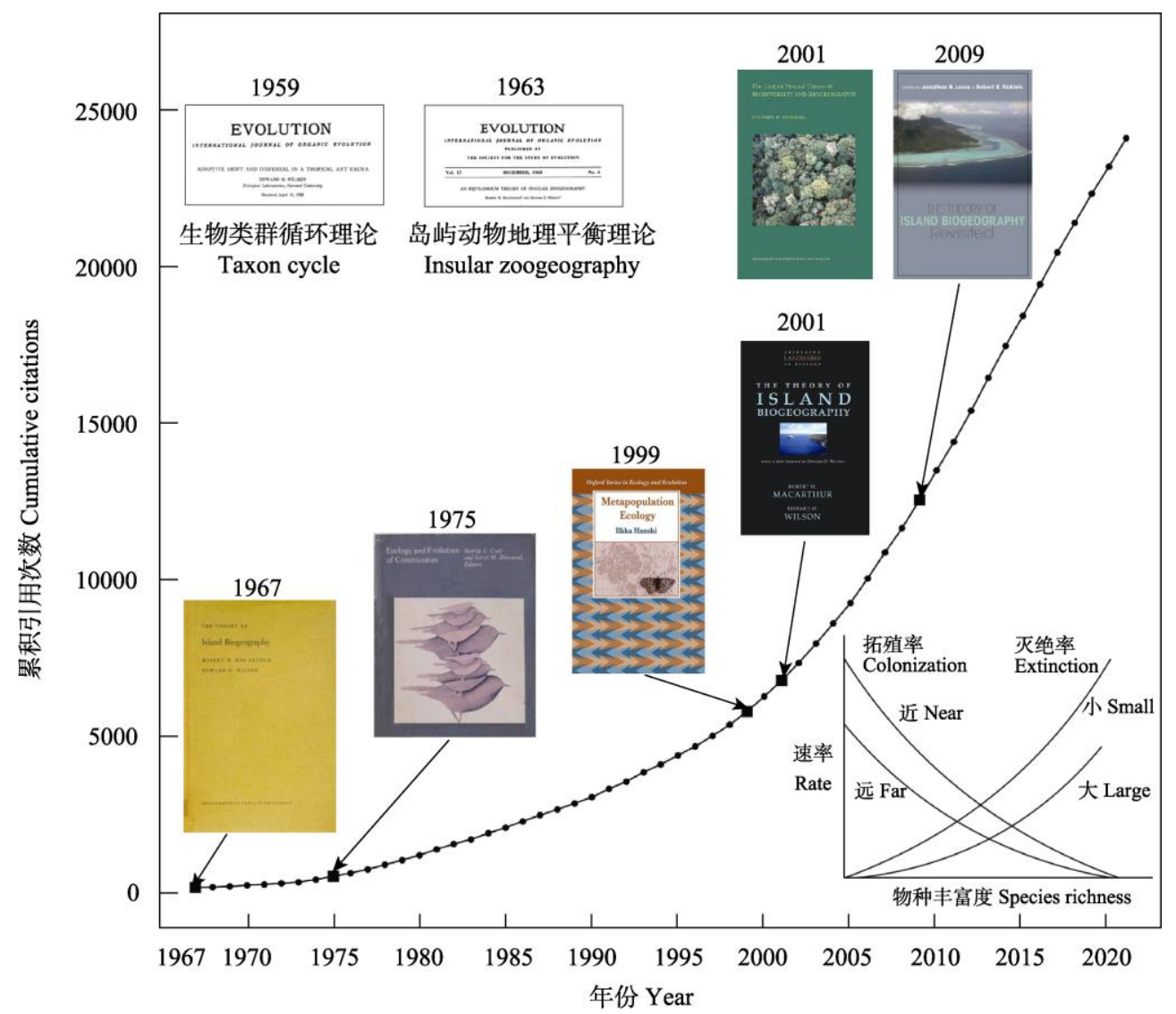

图1《岛屿生物地理学理论》专著的累积引用次数。图示重要前期理论基础、后续纪念专辑与衍生理论(如: 集合种群理论、 群落生态学的中性理论)。

Fig. 1 The cumulative citations of The Theory of Island Biogeography. In this figure, two important foundation works of this theory, the memorial monographs, and the derived theories (e.g., metapopulation theory, and the neutral theory in community ecology) are highlighted.

种重新拓殖的过程。第一次选择的地点是地处墨西 哥湾的干龟群岛(Dry Tortugas)。1965年夏天, 他与 研究生系统调查了岛礁, 在上面发现了为数不多的 植物和节肢动物。他们的想法是让现状继续, 等待 风具风完全清除了岛屿生物后, 跟踪观察植物和节肢 动物的重新拓殖过程。如有神助, 当年就有两次风具 风席卷了干龟群岛。当1966年返回调查时发现, 几 个月前观察的岛礁没有了陆地生物。这样, 他们的 研究就开始了。

然而, 威尔逊对这个实验的前景越来越不满 意。因为岛屿和动植物群落太少, 风具风太少也难以 预测, 并且无法控制。于是, 他又去了佛罗里达湾 的红树植物岛(Mangrove Islet), 这里没有干龟群岛 的缺点, 但有其他不足, 那就是风县风不可能把所有 的节肢动物都从茂密的红树林叶子上带走(Wilson, 2009)。
威尔逊与博士生丹尼尔选择了位置最有利和 最适合的红树植物岛, 请专业的灭虫专家史蒂 夫. 坦德里希(Steve Tendrich)在不损害植被的情况 下, 使用甲基溴熏蒸法 “去除”节肢动物。之后, 丹尼 尔开始每月对每个红树植物岛逐厘米进行检查的 艰苦过程, 同时, 在分类学家的帮助下, 将这些小 岛上的甲虫、树皮虫、蛾类、蜘蛛、螨虫和其他节 肢动物分类到种水平(Simberloff \& Wilson, 1969)。

两年内, 所有红树植物岛上的物种数量差不多 恢复到了灭绝前的水平。如预期的那样, 最遥远的 红树植物岛一开始的物种数很少, 后来又恢复到原 来的低水平, 从而证明了物种平衡的存在。然而, 令人惊讶的是, 红树植物岛间物种组成存在差异, 同一岛上的物种在灭绝前后也存在差异(Simberloff \& Wilson, 1971)。此外, 大多数物种重新定居的速 度和广泛而频繁的周转, 也基本符合麦克阿瑟-威 
尔逊平衡模型。

\section{岛屿生物地理学理论: 科学革命的典事}

麦克阿瑟和威尔逊的物种平衡理论强烈地影 响了生物地理学、生态学和进化生物学等相关理论 的科学思维(Kuhn, 1996)。岛屿生物地理学理论解决 了20世纪该领域的前范式困境, 即, 由独立个案到 多时期和多站点, 以及从扩散论向起源-扩散-适应 中心思想转变, 这些思想达到了生物地理学传统静 态理论的极限。作为回应, 大胆而富有远见的威尔 逊与麦克阿瑟开创性地提出了生物类群循环理论 和岛屿物种平衡理论, 从而化解了这场科学危机。

麦克阿瑟和威尔逊的物种平衡理论之所以能 够得到迅速和压倒性的赞誉, 在很大程度上可归功 于3个因素：(1)相对简单性和由此产生的美学吸引 力; (2)及时性, 即, 在具备必要的科学工具的情况 下取得先进性; (3)真实、协作性质，即，现实性。

岛屿生物地理学理论提出后, 我们关于多尺度 生物地理过程的理解取得了巨大进步(图1), 在理论 方面, 为分析和解释宏观群落提供了生态过程新模 型(Cody \& Diamond, 1975), 并衍生形成了集合种 群理论 (Hanski， 1999)和中性理论 (Hubbell, 2001), 在实践方面, 有效地推动了生物多样性保护和自然 保护地规划(Diamond et al, 1976)。2017年, 在The Theory of Island Biogeography发表50年之际，世界 各地的生态学家和生物地理学家通过各种方式纪 念这一伟大成果对生态学和进化学等领域的贡献。 2016年的国际岛屿生物学大会在总结该领域50年 来发展成果的基础上，通过问卷调查，系统梳理了 岛屿生物地理学末来的发展路线图, 遴选出了50个 亟需重点解决的科学问题(Patiňo et al, 2017)。可以 预见, 生物地理学正在经历一波新的范式转变, 或 者更恰当地说是范式整合 (Whittaker \& Fernández-Palacios, 2007; Losos \& Ricklefs, 2009; Warren et al, 2015; Whittaker et al, 2017)。但无论如 何, 新的范式应该与威尔逊的原始理论共享一些基 本属性(Pickett et al, 2007; Lomolino \& Brown, 2009)。

\section{ORCID}

阎恩荣 (D) https://orcid.org/0000-0002-8064-3334 斯幸峰 (iD https://orcid.org/0000-0003-4465-2759
张健 (iD https://orcid.org/0000-0003-0589-6267

陈小勇 (iD https://orcid.org/0000-0002-4795-8940

\section{参考文献}

Cody ML, Diamond JM (1975) Ecology and Evolution of Communities. Harvard University Press, Cambridge, Massachusetts.

Dammermann KW (1948) The fauna of Krakatau, 1883-1933. Koninklïjke Nederlandsche Akademie Wetenschappen Verhandelingen, 44, 1-594.

Darlington PJ (1948) The geographical distribution of cold-blooded vertebrates. The Quarterly Review of Biology, 23, 1-26.

Darlington PJ (1957) Zoogeography: The Geographical Distribution of Animals. John Wiley \& Sons, New York.

Diamond JM, Terborgh J, Whitcomb RF, Lynch JF, Opler PA, Robbins CS, Simberloff DS, Abele LG (1976) Island biogeography and conservation: strategy and limitations. Science, 193, 1027-1032.

Hanski I (1999) Metapopulation Ecology. Oxford University Press, Oxford.

Hubbell SP (2001) The Unified Neutral Theory of Biodiversity and Biogeography. Princeton University Press, Princeton.

Kuhn TS (1996) The Structure of Scientific Revolutions, 3rd edn. University of Chicago Press, Chicago.

Lack D (1937) The psychological factor in bird distribution. British Birds, 31, 130-136.

Lomolino MV, Brown JH (2009) The reticulating phylogeny of island biogeography theory. The Quarterly Review of Biology, 84, 357-390.

Losos JB, Ricklefs RE (2009) The Theory of Island Biogeography Revisited. Princeton University Press, Princeton.

MacArthur RH, Wilson EO (1963) An equilibrium theory of insular zoogeography. Evolution, 17, 373-387.

MacArthur RH, Wilson EO (1967) The Theory of Island Biogeography. Princeton University Press, Princeton.

Matthew WD (1915) Climate and evolution. Annals of the New York Academy of Sciences, 24, 171-318.

Mayr E (1942) Systematics and the Origin of Species, from the Viewpoint of a Zoologist. Columbia University Press, New York.

Munroe E (1963) Perspectives in biogeography. The Canadian Entomologist, 95, 299-308.

Pickett STA, Kolasa J, Jones CG (2007) Ecological Understanding: The Nature of Theory and the Theory of Nature. Academic Press, Burlinton.

Patiño J, Whittaker RJ, Borges PAV (2017) A roadmap for island biology: 50 fundamental questions after 50 years of the Theory of Island Biogeography. Journal of Biogeography, 44, 963-983.

Simberloff DS, Wilson EO (1969) Experimental zoogeography of islands: Defaunation and monitoring techniques. Ecology, 
50, 267-278.

Simberloff DS, Wilson EO (1971) Experimental zoogeography of islands: A two-year record of colonization. Ecology, 51, 934-937.

Simpson GG (1943) Mammals and the nature of continents. American Journal of Science, 241, 1-31.

Warren BH, Simberloff D, Ricklefs RE, Aguilée R, Condamine FL, Gravel D, Morlon H, Mouquet N, Rosindell J, Casquet J, Conti E, Cornuault J, Fernández-Palacios JM, Hengl T, Norder SJ, Rijsdijk KF, Sanmartín I, Strasberg D, Triantis KA, Valente LM, Whittaker RJ, Gillespie RG, Emerson BC, Thébaud C (2015) Islands as model systems in ecology and evolution: Prospects fifty years after MacArthur-Wilson. Ecology Letters, 18, 200-217.

Wilson EO (1959) Adaptive shift and dispersal in a tropical ant fauna. Evolution, 13, 122-144.

Wilson EO (1961) The nature of the taxon cycle in the
Melanesian ant fauna. The American Naturalist, 95, 169-193.

Wilson EO (1994) Naturalist. Island Press, Washington, DC.

Wilson EO (2009) Island biogeography in the 1960s. The Theory of Island Biogeography Revisited. (eds Losos JB, Ricklefs RE), pp. 1-12. Princeton University Press, Princeton.

Whittaker RJ, Fernández-Palacios JM (2007) Island Biogeography: Ecology, Evolution and Conservation, 2nd edn. Oxford University Press, New York.

Whittaker RJ, Fernández-Palacios JM, Matthews TJ, Borregaard MK, Triantis KA (2017) Island biogeography: Taking the long view of nature's laboratories. Science, 357, eaam8326.

(责任编辑: 周玉荣) 